\title{
Inquérito sorológico e fatores de riscos associados a ovinos soropositivos a Leptospira spp. no estado de Sergipe, Brasil
}

\author{
[Serological survey and risk factors associated with Leptospira spp. seropositivity in sheeps in the \\ state of Sergipe, Brazil]
}

\section{"Artigo Científico/Scientific Article"}

\author{
Huber Rizzo $^{1}$, Lilian Gregory ${ }^{2}$, Jeferson Silva Carvalho $^{2 *}$, Gabriel José Moura Fraga ${ }^{3}$, Fernanda \\ Ramos Santana Santos ${ }^{3}$, Ivy Tasso Gomes ${ }^{2}$, José Wilton Pinheiro Junior ${ }^{1}$, Zenáide Maria de \\ Morais $^{4}$, Silvio Arruda Vasconcellos ${ }^{4}$
}

\author{
${ }^{1}$ Departamento de Medicina Veterinária, Universidade Federal Rural de Pernambuco, Recife-PE, Brasil. \\ ${ }^{2}$ Departamento de Clínica Médica, Faculdade de Medicina Veterinária e Zootecnia, Universidade de São Paulo, São \\ Paulo-SP, Brasil. \\ ${ }^{3}$ Grupo de Iniciação Científica e Extensão em Buiatria (GICEB), Faculdade Pio Décimo, Aracaju-SE, Brasil. \\ ${ }^{4}$ Departamento de Medicina Veterinária Preventiva e Saúde Animal, Faculdade de Medicina Veterinária e Zootecnia, \\ Universidade de São Paulo, São Paulo-SP, Brasil. \\ *Autor para correspondência/Corresponding author: E-mail: hubervet@gmail.com
}

\begin{abstract}
Resumo
O objetivo desse estudo foi determinar a ocorrência e os fatores associados à soropositividade para Leptospira spp. em ovinos do estado de Sergipe, Brasil, a partir de 1.200 amostras coletadas de sessenta propriedades das suas três mesorregiões entre os anos de 2011 e 2012. Foi observada, pela reação de soroaglutinação microscópica, 37,7\% (452/1.200; IC: 0.357-0.396) de ovinos soropositivos com 93,3\% (56) de propriedades com pelo menos um animal positivo. Os sorogrupos reagentes predominantes foram: Sejroe $(32,1 \%)$, Icterohaemorrhagiae $(23,5 \%)$ e Grippotyphosa $(22,4 \%)$, com presença também de reações para Australis $(7,1 \%)$, Pomona (3,8\%), Autumnalis (3,5\%), Panama (3,3\%), Ballum $(1,8 \%)$, Andamana $(1,3 \%)$, Hebdomadis $(0,9 \%)$ e Seranga $(0,4 \%)$. As titulações variaram de 100 a 3.200 , sendo que $77,2 \%$ delas $\leq 400$. Os fatores associados à presença de ovinos soropositivos a Leptospira spp. foram: criações de subsistência $(\mathrm{p}=0,000, \mathrm{OR}=3,08, \mathrm{IC} 95 \%=1,99-4,78)$ e com finalidade de produção de matrizes e reprodutores $(\mathrm{p}=0,000$, $\mathrm{OR}=2,19$, IC95\%=1,46-3,28). As infecções por Leptospira spp. estão ostensivamente presentes nos rebanhos ovinos do estado de Sergipe, sendo necessária a adoção de práticas de manejo adequadas, direcionadas ao controle de roedores e criação consorciada com bovinos que podem levar a contaminação dos alimentos e pastagens.
\end{abstract}

Palavras-chave: Grippotyphosa; Hardjo; Icterohaemorrhagiae; leptospirose; teste de soroaglutinação microscópica.

\begin{abstract}
The objective of this study was to determine the occurrence and factors associated with Leptospira spp. infection in sheep herds in the state of Sergipe, Northeastern Brazil, on the basis of the analysis of 1,200 serum samples collected from 60 properties of the three mesoregions of the state in 2011-2012. The diagnosis was made by microscopic agglutination test and revealed that $37.7 \%$ sheep (452/1.200; CI: 0.357-0.396) were seropositive and that $93.3 \%(56 / 60)$ of the properties had at least one infected sheep. The most prevalent serogroups were Sejroe (32.1\%), Icterohaemorrhagiae (23.5\%), and Grippotyphosa (22.4\%); although the studied animals were reactive to other serogroups: Australis (7.1\%), Pomona (3.8\%), Autumnalis (3.5\%), Panama (3.3\%), Ballum (1.8\%), Andamana (1.3\%), Hebdomadis $(0.9 \%)$, and Seranga (0.4\%). The titrations ranged from 100 to 3,200, and $77.2 \%$ of animals had titers $\leq 400$. The risk factors associated with the presence of Leptospira spp. seropositive sheep were herds raised for subsistence and reproduction. Infections with Leptospira spp. are
\end{abstract}


present in sheep in the state of Sergipe, and it is necessary to adopt adequate management practices, mainly directed toward control of rodents and intercropping with cattle that can lead to contamination of food and pasture.

Keywords: Grippotyphosa; Hardjo; Icterohaemorrhagiae; leptospirosis; microscopic agglutination test.

\section{Introdução}

A leptospirose ovina é uma infecção cosmopolita estritamente vinculada a fatores ambientais, principalmente em propriedades que adotam atividades consorciadas com outras espécies animais (Escócio et al., 2010). Os ovinos adquirem a infecção através da água, contato direto ou indireto com urina, fluidos vaginais, placenta e sêmen infectado, coito ou infecção intrauterina, sendo dentre os ruminantes domésticos, a espécie menos susceptível, podendo tornar-se portadores assintomáticos com infecção renal crônica e leptospirúria persistente mantendo a infecção no rebanho com grande potencial zoonótico (Melo et al., 2010).

Os fatores de risco observados para a ocorrência de ovinos soropositivos a Leptospira spp., nos rebanhos nacionais são plantéis com mais de 48 animais ( $\mathrm{p}=0,021 ; \quad \mathrm{OR}=2,26)$, participação em feiras e exposições $(\mathrm{p}=0,055$; $\mathrm{OR}=9,05$ ) (Alves et al., 2012), fornecimento de água de lagoa e/ou pequenas barragens $(\mathrm{p}=0,01$; $\mathrm{OR}=12,95$ ) (Carvalho et al., 2014), presença de felinos para combate de roedores $(\mathrm{p}=0,018$; $\mathrm{OR}=2,15$ ) (Rizzo et al., 2014) e a presença de roedores, felinos, caprinos, suínos $(\mathrm{p}=0,047$; $\mathrm{OR}=0,44)$ e animais silvestres $(\mathrm{p}=0,0001)$ (Pereira et al., 2018).

$\mathrm{Na}$ região Nordeste, em levantamentos realizados nos estados da Bahia, Paraíba, Rio Grande do Norte e Maranhão, a ocorrência de ovinos soropositivos pelo teste de soroaglutinação microscópica (MAT), variou entre $3,5 \%$ e 94,43\%. Os principais sorogrupos evidenciados foram: Autumnalis, Ballum, Canicola, Icterohaemorrhagiae, Pomona, Tarassovi e Sejroe na Bahia (Viegas et al., 1980; Caldas et al., 1983; Viegas et al., 1994; Melo et al., 2010), Autumnalis, Andamana, Ballum e Djasiman na Paraíba (Higino et al., 2010; Alves et al., 2012; Costa et al., 2016), Ballum, Autumnalis e Pomona no Rio Grande do Norte (Azevedo et al., 2004) e Grippotyphosa, Sentot, Hardjo e Andanda no Maranhão (Carvalho et al., 2014; Pereira et al., 2018).

Nas demais regiões do Brasil existem levantamentos sorológicos nos estados de
Rondônia, Pará, São Paulo, Rio de Janeiro e Rio Grande do Sul, além do Distrito Federal com ocorrências de ovinos soropositivos entre 3\% e 47,4\% (Silva et al., 2007; Aguiar et al., 2010; Seixas et al., 2011; Martins et al., 2012; Moraes et al., 2012; Amorim et al., 2016).

Devido à variabilidade de resultados no Brasil e à inexistência de estudos no estado de Sergipe, a presente pesquisa teve por objetivo determinar a ocorrência e os fatores de risco associados à presença de aglutininas antiLeptospira spp. em seus rebanhos ovinos.

\section{Material e Métodos}

O inquérito sorológico foi realizado no estado de Sergipe $\left(9^{\circ} 30^{\prime} 49^{\prime \prime}\right.$ e $11^{\circ} 34^{\prime} 05^{\prime \prime}$ latitude; $36^{\circ} 23^{\prime} 40^{\prime \prime}$ e $38^{\circ} 15^{\prime} 00^{\prime \prime}$ longitude) que possui área territorial de $21.915 \mathrm{Km}^{2}$, dividida em três mesorregiões edomorfoclimáticas com clima tropical no litoral e semiárido no interior. Possui $1,4 \%$ do rebanho de ovinos da região Nordeste, com um total de 136.269 cabeças, distribuídas nas três mesorregiões (51,5\% no agreste, $26 \%$ no sertão e 22,5\% no leste) (IBGE, 2014).

O cálculo amostral foi realizado conforme Thrusfield (1995) e teve como base a população de 136.269 ovinos (IBGE, 2017), nível de confiança de $95 \%$ e percentual de margem de erro amostral de 5\%, utilizando a prevalência de $\pm 36,17 \%$ (Viegas et al., 1980; Caldas et al., 1983; Viegas et al., 1994; Azevedo et al., 2004; Higino et al., 2010; Melo et al., 2010; Alves et al., 2012; Carvalho et al., 2014; Costa et al., 2016; Pereira et al., 2018), resultando em amostragem mínima de 369 animais sendo 123 por mesorregião.

As 1.2000 amostras de sangue ovinos foram coletadas, entre março de 2011 e dezembro de 2012, de sessenta propriedades de dezenove municípios das três mesorregiões do estado: leste, agreste e sertão, totalizando de 680, 280 e 240 amostras respectivamente (Figura 1).

As propriedades utilizadas no estudo foram selecionadas por conveniência, a partir da facilidade de acesso e disponibilidade dos produtores, onde coletou-se aleatoriamente vinte animais por rebanho, de diferentes padrões zootécnicos, aparentemente hígidos, com idade 
superior a seis meses e não vacinados contra leptospirose. Os criadores foram submetidos a um questionário epidemiológico com 34 perguntas relacionadas às características de manejo e instalações dos rebanhos, a fim de avaliar os fatores associados à presença de Leptospira spp.

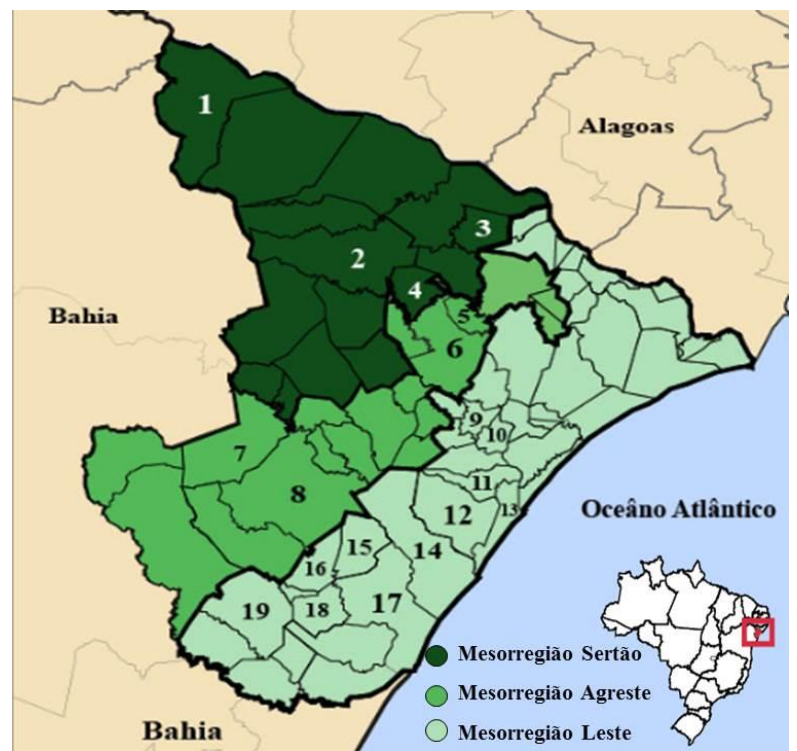

Figura 1. Mapa de Sergipe dividido entre suas três mesorregiões (leste, agreste e sertão) e demonstrando os munícipios de coleta para a pesquisa de aglutininas anti-Leptospira em ovinos, com numeração correspondente ao nome do município. Região sertão: 1-Canindé de São Francisco, 2-Nossa Senhora da Glória, 3-Itabi e 4-Feira Nova. Região agreste: 5Cumbe, 6-Nossa Senhora das Dores, 7-Simão Dias e 8-Lagarto. Região leste: 9-Divina Pastora, 10-Maruim, 11-Nossa Senhora do Socorro, 12-São Cristóvão, 13-Aracaju, 14Itaporanga D'Ajuda, 15-Salgado, 16-Boquim, 17-Estância, 18-Arauá e 19-Itabaianinha (IBGE, 2014).

As amostras de sangue foram coletadas em tubos estéreis a vácuo, por punção da veia jugular, centrifugadas a $1.600 \times \mathrm{g}$ por dez minutos, acondicionadas em tubos de polipropileno de 2 $\mathrm{mL}$ e congeladas a $-20^{\circ} \mathrm{C}$, até o processamento. $\mathrm{O}$ MAT, para detecção de anticorpos contra Leptospira spp., foi realizado no Laboratório de Zoonoses Bacterianas da Faculdade de Medicina Veterinária e Zootecnia da Universidade de São Paulo utilizando coleção de 26 sorovares (Leptospira interrogans sorovars Australis, Bataviae, Bratislava, Butembo, Canicola, Copenhageni, Fronn, Grippotyphosa, Hebdomadis, Icterohaemorrhagiae, Pomona, Pyrogenes, Sentot, Whitcombi e Wolffi; Leptospira borgpeterseni sorovars Autumnalis, Castellonis, Hardjo, Hardjobovis Javanica, Mini e Tarassovi; Leptospira santarosai sorovars
Shermani; Leptospira kirschneri sorovars Cynopteri; Leptospira noguchii serovars Panama e Leptospira biflexa sorovars Andamana e Patoc) pertencentes a 21 sorogrupos, sendo as leptospiras cultivadas no meio EMJH modificado (Alves et al., 1996). As amostras com aglutinação evidente na diluição 1:100 foram consideradas positivas (Faine et al., 1999).

As determinações das frequências, absolutas e relativas, foram obtidas através da análise estatística descritiva. Para o estudo dos fatores de risco, foi realizada a análise univariada das variáveis de interesse pelo teste qui-quadrado de Pearson, ou exato de Fisher, quando necessário. Posteriormente, foi realizada a análise de regressão logística considerando como variável dependente para a infecção por Leptospira spp. o resultado do MAT (positivo ou negativo). As variáveis independentes ou explanatórias consideradas no modelo foram aquelas que apresentaram significância estatística $<0,20$. Essa probabilidade foi estipulada para que possíveis fatores de risco do evento não fossem excluídos da análise (Hosmer e Lemeshow, 1989). O programa EpiInfo 7.2.2.6 foi utilizado para a execução dos cálculos estatísticos.

\section{Resultados e Discussão}

No presente estudo a ocorrência de ovinos soropositivos a Leptospira spp. foi de $37,7 \%$ (452/1.200; CI: 0.357-0.396), estando presente em 93,3\% (56/60) das propriedades e em todos os municípios pesquisados (Tabela 1).

A taxa obtida coloca Sergipe, entre os levantamentos realizados na região Nordeste, atrás de estudos da Bahia entre os anos de 1991 e 1998 com soropositividade entre $46 \%$ e $89,5 \%$, mas supera os demais do mesmo estado, nos anos de 1980, 1983 e 1990, com taxas entre $11,7 \%$ e 22,9\% (Viegas et al., 1994; Melo et al., 2010) e do recente levantamento realizado no Maranhão com $94,43 \%$. Outros levantamentos da região, com taxas abaixo das desse estudo, foram realizadas no Rio Grande do Norte (3,5\%) (Azevedo et al., 2004), Maranhão (31,9\%) (Carvalho et al., 2014) e três na Paraíba $(5,4 \%, 7,5 \%$ e $11,2 \%$ ) (Higino et al., 2010; Alves et al., 2012; Costa et al., 2016).

Estudos realizados na Paraíba com ovinos de abatedouros apresentaram baixas taxas, sendo justificado pelo período seco do ano no qual as amostras foram coletadas, ambiente desfavorável para a manutenção de leptospiras, ou rusticidade do rebanho, contrariando o realizado no presente 
estudo onde as amostras foram coletadas durante todos os meses do ano e $94,7 \%$ dos animais apresentavam padrão racial definido, em grande maioria Santa Inês. A questão racial pode ser um ponto importante na infecção e manutenção da titulação contra Leptospira spp., pois Costa et al. (2018) observaram maior susceptibilidade de ovinos Santa Inês em relações a animais mestiços, apresentando maiores valores e duração dos títulos, além das reações de positividades serem detectadas mais brevemente após a inoculação experimental via conjuntiva do sorogrupo Pomona.

Tabela 1. Ocorrência de ovinos soropositivos para Leptospira spp. pelo teste de aglutinação microscópica (MAT) em relação ao município e mesorregião do estado de Sergipe, Brasil, 2011-2012

\begin{tabular}{|c|c|c|}
\hline Município & \% (Propriedades com positivo/Total) & $\%$ (Soropositivos/Total) \\
\hline Aracaju & $100(1 / 1)$ & $20(4 / 20)$ \\
\hline Arauá & $100(3 / 3)$ & $43,3(26 / 60)$ \\
\hline Boquim & $100(1 / 1)$ & $85(17 / 20)$ \\
\hline Divina Pastora & $100(3 / 3)$ & $91,7(55 / 60)$ \\
\hline Estância & $75(3 / 4)$ & $17,5(14 / 80)$ \\
\hline Itabaianinha & $100(9 / 9)$ & $46,1(83 / 180)$ \\
\hline Itaporanga D'Ajuda & $100(5 / 5)$ & $43(43 / 100)$ \\
\hline Maruim & $100(2 / 2)$ & $60(24 / 40)$ \\
\hline Nossa Senhora do Socorro & $100(1 / 1)$ & $50(10 / 20)$ \\
\hline Salgado & $100(3 / 3)$ & $28,3(17 / 60)$ \\
\hline São Cristóvão & $100(2 / 2)$ & $37,5(15 / 40)$ \\
\hline Mesorregião Leste & $97,1(33 / 34)$ & $45,3(308 / 680)$ \\
\hline Cumbe & $100(1 / 1)$ & $30(6 / 20)$ \\
\hline Lagarto & $100(6 / 6)$ & $55(66 / 120)$ \\
\hline Nossa Senhora das Dores & $75(3 / 4)$ & $30(24 / 80)$ \\
\hline Simão Dias & $100(3 / 3)$ & $20(12 / 60)$ \\
\hline Mesorregião Agreste & $92,9(3 / 14)$ & $38,6(108 / 280)$ \\
\hline Canindé de São Francisco & $75(3 / 4)$ & $15(12 / 80)$ \\
\hline Feira Nova & $100(2 / 2)$ & $22,5(9 / 40)$ \\
\hline Itabi & $100(2 / 2)$ & $17,5(7 / 40)$ \\
\hline Nossa Senhora da Glória & $75(3 / 4)$ & $10(8 / 80)$ \\
\hline Mesorregião Sertão & $83,3(10 / 12)$ & $15(36 / 240)$ \\
\hline Total & $93,3(56 / 60)$ & $37,7(452 / 1200)$ \\
\hline
\end{tabular}

A influência do clima foi notada quando avaliamos os resultados obtidos por mesorregião onde o sertão (15\%), com o clima mais seco do estado, apresentou menor taxa de ovinos soropositivos que as outras duas, agreste $(38,6 \%)$ e leste $(45,3 \%)$, que possuem maiores índices pluviométricos e clima mais úmido $(\mathrm{p}=0,000)$, uma vez que o contato estreito entre animais excretores, criados sob pastejo num habitat com elevada retenção de água é limitante para o ciclo de transmissão interespécie ou intraespécie (Escócio et al., 2010).

Foram observadas reações de soroaglutinação contra onze sorogrupos sendo os mais predominantes o Sejroe, Icterohaemorrhagiae e Grippotyphosa que concentraram $77,88 \%$ das reações, complementado por Australis, Pomona, Autumnalis, Panama, Ballum, Andamana, Hebdomadis e Seranga que representaram os outros 22,12\% de reações (Tabela 2).
O sorogrupo Sejroe é o maior responsável pelas perdas reprodutivas em bovinos e também causador de abortamentos nos ovinos, sendo que a elevada presença de reações contra esse sorogrupo pode estar relacionada com criação consorciada entre essas espécies, que foi relatada em $85 \%$ (51/60) das propriedades, assim como descreveu Escócio et al. (2010) em São Paulo. No entanto, ovinos podem servir como hospedeiro de manutenção desse sorogrupo sugerindo a ocorrência de transmissão ativa dentro do plantel por contato direto ou indireto (Melo et al., 2010).

Outros importantes sorogrupos foram o Grippotyphosa, o mais reativo no Maranhão (Carvalho et al., 2014), e Icterohaemorrhagiae que destacam a necessidade da inclusão de programas de desratização junto às propriedades uma vez que os principais hospedeiros de manutenção de ambos são o rato (Rattus norvegivus), onde a presença desses reservatórios, indica as deficientes práticas sanitárias, tais como: o acúmulo de lixo e resíduos orgânicos, falta de 
higiene nos comedouros e bebedouros, mau armazenamento dos alimentos, negligência com a qualidade da água, proliferação de animais sinantrópicos e silvestres de vida livre em torno das propriedades, estreitando seu contato com animais domésticos e o homem (Silva et al., 2014). O sorogrupo Autumnalis, que possui alta frequência nos estudos da região Nordeste (Azevedo et al., 2004; Higino et al., 2010; Melo et al., 2010; Alves et al., 2012; Pereira et al., 2018) e geralmente associado à presença de animais silvestres e criatórios próximos a reservas florestais, apresentou baixa ocorrência nos rebanhos Sergipanos $(2,66 \%)$, mesmo com $80 \%$ dos criadores relatando a circulação de animais silvestres no entorno das propriedade.

Tabela 2. Distribuição decrescente das 452 reações e titulações de aglutininas anti-Leptospira pelo teste de aglutinação microscópica (MAT) em ovinos criados no estado de Sergipe, Brasil, 2011-2012

\begin{tabular}{|c|c|c|c|c|c|c|c|}
\hline \multirow[t]{2}{*}{ Sorogrupo } & \multicolumn{6}{|c|}{ Titulação (\%) } & \multirow{2}{*}{ Total (\%) } \\
\hline & 100 & 200 & 400 & 800 & 1600 & 3200 & \\
\hline Sejroe & $6(1,3)$ & $45(10)$ & $43(9,5)$ & $33(7,3)$ & $16(3,5)$ & $2(0,4)$ & $145(32,1)$ \\
\hline Icterohaemorrhagiae & $59(13,1)$ & $35(7,7)$ & $11(2,4)$ & - & $1(0,2)$ & - & $106(23,5)$ \\
\hline Grippotyphosa & $4(0,9)$ & $25(5,5)$ & $34(7,5)$ & $30(6,7)$ & $7(1,6)$ & $1(0,2)$ & $101(22,4)$ \\
\hline Australis & $6(1,3)$ & $12(2,7)$ & $7(1,6)$ & $6(1,3)$ & $1(0,2)$ & - & $32(7,1)$ \\
\hline Pomona & $10(2,2)$ & $6(1,3)$ & $1(0,2)$ & - & - & - & $17(3,8)$ \\
\hline Autumnalis & $3(0,7)$ & $5(1,1)$ & $4(0,9)$ & $4(0,9)$ & - & - & $16(3,5)$ \\
\hline Panama & $2(0,4)$ & $5(1,1)$ & $6(1,3)$ & $2(0,4)$ & - & - & $15(3,3)$ \\
\hline Ballum & $4(0,9)$ & $4(0,9)$ & - & - & - & - & $8(1,8)$ \\
\hline Andamana & $2(0,4)$ & $4(0,9)$ & - & - & - & - & $6(1,3)$ \\
\hline Hebdomadis & $1(0,2)$ & $1(0,2)$ & $2(0,4)$ & - & - & - & $4(0,9)$ \\
\hline Seranga & $1(0,2)$ & $1(0,2)$ & - & - & - & - & $2(0,4)$ \\
\hline Total & $98(21,6)$ & $143(32,6)$ & $108(23,8)$ & $75(16,5)$ & $25(5,5)$ & $3(0,6)$ & $452(100)$ \\
\hline
\end{tabular}

É importante a análise da ocorrência dos sorogrupos na região estudada devido à preconização futura de vacinação, caso seja necessária em situações de perdas reprodutivas, visto que não há imunidade cruzada e as vacinas disponíveis são compostas pelos sorogrupos Canicola, Grippotyphosa, Sejroe, Icterohaemorrhagiae e Pomona (Azevedo et al., 2004), agravando-se nos rebanhos estudados uma vez que nenhum criador realizava vacinação preventiva contra o agente.

Quanto às titulações, estas variaram de 100 a 3.200 sendo que $77,21 \%$ (349) delas $\leq 400$ (Tabela 2). A ocorrência de baixas titulações em ovinos demonstra que a leptospirose é uma doença comum e provavelmente subestimada devido à grande quantidade de animais assintomáticos. Foram poucos ovinos detectados com altos títulos para os diversos tipos de sorogrupos estudados, o que demonstra que apesar da bactéria estar circulando em diversas espécies ela não vem causando, em ovinos, manifestações graves relacionadas a perdas reprodutivas.

Após análise univariada (Tabela 3) e de regressão logística, confirmaram-se como fatores de risco para a presença de ovinos com aglutininas anti-Leptospira criações com finalidade de subsistência $(\mathrm{p}=0,000, \mathrm{OR}=3,08$, IC95\%=1,99-
$4,78)$ e de reprodução $(\mathrm{p}=0,000, \quad \mathrm{OR}=2,19$, IC95\%=1,46-3,28) visando à venda de matrizes e reprodutores.

As criações de subsistência, que possuíam baixa tecnificação, eram constituídas por poucos animais mestiços ou da raça Santa Inês, contrapondo os achados de Alves et al. (2012) que observaram como risco rebanhos com mais de 48 ovinos ( $\mathrm{p}=0,021$ e $\mathrm{OR}=2,26)$ sugerindo que a aglomeração de ovinos os expõe a fômites, urina, secreções vaginais e abortos com maior incidência.

Essas aglomerações ou confinamentos, devido ao sistema de criação intensivo ou semiextensivo, foram observados em propriedades com o objetivo de produção de matrizes e reprodutores em Sergipe, onde os animais permaneciam mais tempo em apriscos, embaiados ou em pequenos piquetes. Outro fator que pode estar relacionado com a infecção, nesse tipo de criação, é a circulação desses animais em aglomerações como feiras e exposições, como foi observado na Paraíba ( $\mathrm{p}=0,055$ e $\mathrm{OR}=9,05$ ) (Alves et al., 2012). Junta-se a esses fatores a predominância nessas propriedades de animais de raças puras (Santa Inês e Dorper) de baixa rusticidade, que possam ser mais susceptíveis ao agente (Costa et al., 2016; 2018). 
Tabela 3. Ocorrência de ovinos soropositivos para Leptospira spp. pelo teste de aglutinação microscópica (MAT), oriundos de propriedades rurais do estado de Sergipe, por variável analisada e total, 2011-2012

\begin{tabular}{|c|c|c|c|c|}
\hline Variável & $\mathbf{N}$ & MAT (\%) & OR (IC 95\%) & Valor de p \\
\hline \multicolumn{5}{|l|}{ Microrregião } \\
\hline Leste & 680 & $308(45,3)$ & - & \multirow{3}{*}{0,000} \\
\hline Agreste & 280 & $108(38,6)$ & $0,76(0,56-1,02)$ & \\
\hline Sertão & 240 & $36(15)$ & $0,28(0,18-0,44)$ & \\
\hline \multicolumn{5}{|l|}{ Sexo } \\
\hline Fêmea & 990 & $391(39,5)$ & \multirow[t]{2}{*}{$1,59(1,14-2,24)$} & \multirow[t]{2}{*}{0,002} \\
\hline Macho & 210 & $61(29)$ & & \\
\hline \multicolumn{5}{|l|}{ Raça } \\
\hline Dorper & 72 & $24(33,3)$ & - & 0,243 \\
\hline Santa Inês & 1064 & $413(38,8)$ & $1,476(1,003-2,172)$ & 0,028 \\
\hline Mestiço & 64 & $15(24,2)$ & $0,506(0,280-0,916)$ & 0,014 \\
\hline \multicolumn{5}{|l|}{ Finalidade de criação } \\
\hline Cria/recria/engorda & 960 & $322(33,5)$ & - & \multirow{3}{*}{0,000} \\
\hline Reprodução & 140 & $67(47,9)$ & $1,82(1,25-2,64)$ & \\
\hline Subsistência & 100 & $63(63)$ & $1,86(1,06-3,25)$ & \\
\hline \multicolumn{5}{|l|}{ Regime de criação } \\
\hline Extensivo & 500 & $241(48,2)$ & - & \multirow[t]{3}{*}{0,000} \\
\hline Intensivo & 60 & $6(10)$ & $0,12(0,04-0,28)$ & \\
\hline Semiextensivo & 640 & $205(32)$ & $4,24(1,78-12,24)$ & \\
\hline \multicolumn{5}{|l|}{ Instalações } \\
\hline Aprisco de alvenaria & 120 & $56(46,7)$ & - & \multirow[t]{3}{*}{0,067} \\
\hline Aprisco de chão batido & 540 & $191(35,4)$ & $0,63(0,41-0,95)$ & \\
\hline Aprisco de piso ripado & 420 & $160(38,1)$ & $1,12(0,86-1,48)$ & \\
\hline \multicolumn{5}{|l|}{ Fonte de água } \\
\hline Corrente & 220 & $90(40,9)$ & - & \multirow[t]{3}{*}{0,000} \\
\hline Parada & 620 & $158(25,6)$ & $0,49(0,35-0,69)$ & \\
\hline Parada e corrente & 360 & $204(56,7)$ & $3,82(2,87-5,09)$ & \\
\hline \multicolumn{5}{|c|}{ Circulação de animais silvestres } \\
\hline $\operatorname{Sim}$ & 960 & $396(41,3)$ & $2,30(1,67-3,21)$ & 0,000 \\
\hline Não & 240 & $56(23,3)$ & & \\
\hline
\end{tabular}

\section{Conclusão}

Leptospira spp. estão ostensivamente presentes nos rebanhos ovinos do estado de Sergipe, relacionadas principalmente à finalidade de criação, tendo como sorogrupos prevalentes Sejroe, Icterohaemorrhagiae e Grippotyphosa, que indicam a adoção de medidas de controle nas propriedades voltadas principalmente a evitar a criação consorciada com bovinos e a presença de roedores que contaminam os alimentos, sendo a via oral importante porta de entrada do agente.

\section{Conflito de Interesse} interesse.

Os autores declaram não existir conflito de

\section{Comitê de Ética}

$\mathrm{O}$ projeto de pesquisa foi aprovado pelo comitê de ética da Faculdade Pio Décimo, sob o número 06/2011.

\section{Agradecimentos}

Ao Grupo de Iniciação Científica e Extensão em Buiatria (GICEB) da Faculdade Pio Décimo de Aracaju/SE pela execução do Projeto.

\section{Referências}

Aguiar, D.M.; Cavalcante, G.T.; Marvulo, M.F.V.; Silva, J.C.R.; Pinter, A.; Vasconcelos, S.A.; Morais, Z.M.; Labruna, M.B.; Camargo, L.M.A.; Gennari, S.M. Anticorpos anti-Leptospira spp. em ovinos do município de Monte Negro, estado de Rondônia. Arquivos do Instituto Biológico, 77(3): 529-532, 2010.

Alves, C.J.; Vasconcelos, S.A.; Camargo, C.R.A.; Morais, Z.M. Influência de fatores ambientais sobre a proporção de caprinos soro-reatores para a leptospirose em cinco centros de criação do Estado da Paraíba, Brasil. Arquivos do Instituto Biológico, 63(2): 11-18, 1996.

Alves, C.J.; Alcindo, J.F.; Farias, A.E.M.; Higino, S.S.S.; Santos, F.A.; Azevedo, S.S.; Costa, D.F.; Santos, C.S.A.B. Caracterização 
epidemiológica e fatores de risco associados à leptospirose em ovinos deslanados do semiárido brasileiro. Pesquisa Veterinária Brasileira, 32(6): 523-528, 2012.

Amorim, R.M.; Nascimento, E.M.; Santa Rosa, B.P.; Dantas, G.N.; Ferreira, D.O.L.; Gonçalves, R.C.; Ulmann, L.S.; Langoni, H. Soroprevalência da leptospirose em ovinos da região Centro-Oeste do estado de São Paulo. Veterinária e Zootecnia, 23(2): $297-$ 305, 2016.

Azevedo, S.S.; Alves, C.J.; Andrade, J.S.L.; Batista, C.S.A.; Clementino, I.J.; Santos, F.A. Ocorrência de aglutininas antiLeptospira em ovinos do estado do Rio Grande do Norte, Brasil. Revista Brasileira de Ciência Veterinária, 11(3): 167-170, 2004.

Caldas, E.M.; Sampaio M.B.; Viegas, E.A.; Viegas, S.A.R.A.; Dias, E.M.M. Aglutininas anti-Leptospira em ovinos e caprinos na região Nordeste do Estado da Bahia. Arquivos da Escola de Veterinária da Universidade Federal da Bahia, 8(11): 8889, 1983.

Carvalho, S.M.; Mineiro, A.L.; Castro, V.; Genovez, M.E.; Azevedo, S.S.; Costa, F.A. Leptospirosis seroprevalence and risk factors for sheep in Maranhão state, Brazil. Tropical Animal Health and Production, 46(2): 491494, 2014.

Costa, D.F.; Silva, A.F.; Farias, A.E.M.; Brasil, A.W.L.; Santos, F.A.; Guilherme, R.F.; Azevedo, S.S.; Alves, C.J. Serological study of the Leptospira spp. infection in sheep and goats slaughtered in the State of Paraiba, semiarid of Northeastern Brazil. Semina: Ciências Agrárias, 37(2): 819-828, 2016.

Costa, D.F.; Silva, M.L.C.R.; Martins, G.; Dantas, A.F.M.; Mela, M.A.; Azevedo, S.S.; Lilenbaum, W.; Alves, C.J. Susceptibility among breeds of sheep experimentally infected with Leptospira interrogans Pomona serogroup. Microbial Pathogenesis, 122(1): 79-83, 2018.

Escócio, C.; Genovez, M.E.; Castro, V.; Piatti, R.M.; Gabriel, F.H.L.; Chiebao, D.P.; Azevedo, S.S.; Vieira, S.R.; Chiba, M. Influência das condições ambientais na transmissão da leptospirose entre criações de ovinos e bovinos da região de Sorocaba, SP. Arquivos do Instituto Biológico, 77(3): 371-379, 2010.
Faine, S.; Adler, B.; Bolin, C.; Perolat, P. Leptospira and leptospirosis. $2^{\text {nd }}$ ed. Melbourne: MediSci, 1999. 272p.

Higino, S.S.S.; Azevedo, S.S.; Alves, C.J.; Figueiredo, S.M.; Silva, M.L.C.R.; Batista, C.S.A. Frequência de leptospirose em ovinos abatidos no município de Patos, Paraíba. Arquivos do Instituto Biológico, 77(3): 525-527, 2010.

Hosmer, D.; Lemeshow, S. Applied logistic regression. A Wiley-Interscience Publication, New York: John Wiley \& Sons Inc., 1989. 307p.

IBGE. Instituto Brasileiro de Geografia e Estatística. Censo agropecuário. 2017. Disponível em: <https://sidra.ibge.gov.br >. Acesso em: 05 jun. 2018.

IBGE. Instituto Brasileiro de Geografia e Estatística. Bases cartográficas. 2014. Disponível em: < http://geoftp.ibge.gov.br>. Acesso em: 12 jun. 2017.

Martins, G.; Penna, B.; Hamond, C.; Leite, R.C.; Silva, A.; Ferreira, A.; Brandão, F.; Oliveira, F.; Lilenbaum, W. Leptospirosis as the most frequent infectious disease impairing productivity in small ruminants in Rio de Janeiro, Brazil. Tropical Animal Health and Production, 44: 773-777, 2012.

Melo, L.S.S.; Castro, M.B.; Leite, R.C.; Moreira, É.C.; Melo, C. Principais aspectos da infecção por Leptospira sp. em ovinos. Ciência Rural, 40(5): 1235-1241, 2010.

Moraes, C.C.G.; Guerreiro, A.N.; Kuroda, R.B.S.; Souza, V.A.F.; Meneses, A.M.C.; Vasconcellos, S.A. Inquérito sorológico para leptospirose em rebanhos de ovinos no município de Igarapé-Açu, Estado do Pará. Revista de Ciências Agrárias, 55(1): 58-60, 2012.

Pereira, H.M.; Santos, H.P.; Soares, R.R.; Viana Júnior, F.A.M.; Rocha, T.B.; Soares, D.M.; Cavalcante, C.A. Leptospira spp. in sheep of the state of Maranhão, Brazil: frequency, risk factors and foci mapping. Acta Veterinaria Brasilica, 12(1): 29-34, 2018.

Rizzo, H.; Gregory, L.; Beraldi, F.; Castro, V.; Morais, Z.M.; Vasconcellos, S.A. Soropositividade para leptospirose e desempenho reprodutivo de ovinos de criatórios localizados no estado de São Paulo, Brasil. Revista Brasileira de Medicina Veterinária, 36(3): 244-250, 2014. 
Seixas, L.S.; Melo, C.B.; Leite, R.C.; Moreira, E.C.; Mcmanus, C.M.; Castro, M.B. AntiLeptospira sp. agglutinins in ewes in the Federal District, Brazil. Tropical Animal Health and Production, 43(1): 9-11, 2011.

Silva, E.F.; Brod, C.S.; Cerqueira, G.M.; Bourscheidt, D.; Seyffert, N.; Queiroz, A.; Santos, C.S.; Ko, A.I.; Dellagostin, O.A. Isolation of Leptospira noguchii from sheep. Veterinary Microbiology, 121(1-2): 144149, 2007.

Silva, F.J.; Santos, C.E.P.; Silva, G.C.P.; Santos, R.F.; Curci, V.C.M.; Mathias, L.A. The importance of Leptospira interrogans serovars Icterohaemorrhagiae and Canicola in coastal zone and in Southern fields of Rio Grande do Sul, Brazil. Pesquisa Veterinária Brasileira, 34(1): 34-38, 2014.
Thrusfield, M. Veterinary epidemiology. $2^{\text {nd }}$ ed. Oxford: Blackwell Scientific Publication, 1995. 479p.

Viegas, E.A.; Viegas, S.R.A.; Caldas, E.M. Aglutininas anti-Leptospira em hemosoro de caprinos e ovinos, no Estado da Bahia. Arquivos da Escola de Medicina Veterinária da Universidade Federal da Bahia, 5(1): 20-34, 1980.

Viegas, E.A.; Yanaguita, R.M.; Viegas, S.A.R.A.; Silva, L.A.; Vasconcelos, S.A. Emprego de estirpes de Leptospira biflexa na prova de soroaglutinação microscópica aplicada ao diagnóstico da leptospirose caprina e ovina. Brazilian Journal of Veterinary and Animal Science, 31(1): 25-30, 1994. 